\title{
-NOTES-
}

\section{ON THE SPECTRA OF UNITARY HALF-SCATTERING OPERATORS*}

BY C. R. PUTNAM, Purdue University

1. Introduction. Let $H_{0}$ denote a self-adjoint operator, bounded or unbounded, on a Hilbert space of elements $f, g, \cdots$, and suppose that $H_{0}$ is absolutely continuous. (By the absolute continuity of a self-adjoint operator $H$ with the spectral resolution

$$
H=\int_{-\infty}^{\infty} \lambda d E(\lambda)
$$

is meant that $\|E(\lambda) f\|$ is an absolutely continuous function of $\lambda$ for every element $f$; see Rosenblum [15], Kato [10], Kuroda [12], Putnam [13].) Let $V$ denote a self-adjoint operator for which $H_{1}$, where

$$
H_{1}=H_{0}+V
$$

is self-adjoint and absolutely continuous. The operator $H_{1}$ defined by (2) must be selfadjoint if, for instance, $V$ is bounded; non-trivial conditions assuring the absolute continuity of $H_{1}$ however are not so apparent.

In [3] and [4], Friedrichs considered a special pair $H_{0}$ and $V$, where $H_{0}$ was bounded (and also absolutely continuous) and $V$ was a certain type of integral operator, and showed that if $H_{1}$ is defined by

$$
H_{1}^{\epsilon}=H_{0}+\epsilon V, \quad \epsilon \text { real, }
$$

then, for sufficiently small $\epsilon$, the strong limits

$$
\lim _{t \rightarrow \infty} U_{t}^{\epsilon}=U_{+}^{\epsilon} \text { and } \lim _{t \rightarrow-\infty} U_{t}^{\epsilon}=U_{-}^{\epsilon}, \text { where } U_{t}^{\epsilon}=\exp \left(i t H_{1}^{\epsilon}\right) \exp \left(-i t H_{0}\right),
$$

exist, and $U_{+}^{\epsilon}$ and $U_{-}^{\epsilon}$ are unitary operators satisfying

$$
H_{1}^{\epsilon}=U_{+}^{\epsilon} H_{0} U_{+}^{\epsilon *} \text { and } H_{1}^{\epsilon}=U_{-}^{\epsilon} H_{0} U_{-}^{\epsilon *} \text {. }
$$

In addition, each of the operators $U^{\epsilon}=U_{+}^{\bullet}, U_{-}^{\bullet}$ is analytic in $\epsilon$,

$$
U^{\epsilon}=I+\epsilon U_{1}+\epsilon^{2} U_{2}+\cdots,
$$

and hence satisfies

$$
\left\|U^{e}-I\right\| \rightarrow 0 \text { as } \epsilon \rightarrow 0 .
$$

It is seen that condition (7) implies that

$$
s p\left(U^{\epsilon}\right) \rightarrow 1 \text { as } \epsilon \rightarrow 0,
$$

where $\operatorname{sp}(\mathrm{A})$ denotes the spectrum of an operator $\mathrm{A}$.

*Received June 22, 1961. This research was supported by the United States Air Force through the Air Force Office of Scientific Research of the Air Research and Development Command under Contract No. AF 18(603)-139. Reproduction in whole or in part is permitted for any purpose of the United States Government. 
Later, Rosenblum [15] and Kato [9], [10], showed that if (2) holds, where $H_{0}$ and $H_{1}$ are absolutely continuous and if $V$ is of the trace class (that is, $V$ is completely continuous with eigenvalues $\lambda_{1}, \lambda_{2}, \cdots$ satisfying $\left.\sum\left|\lambda_{i}\right|<\infty\right)$ then the strong limits

$$
U_{+}=\lim _{t \rightarrow \infty} U_{t} \text { and } U_{-}=\lim _{t \rightarrow-\infty} U_{t} \text {, where } U_{t}=\exp \left(i t H_{1}\right) \exp \left(-i t H_{0}\right) \text {, }
$$

exist and are unitary operators satisfying

$$
H_{1}=U_{+} H_{0} U_{+}^{*} \text { and } H_{1}=U_{-} H_{0} U_{-}^{*} \text {. }
$$

Similar problems, including that of the perturbation of absolutely continuous spectra, as well as generalizations to cases where $H_{0}$ and $H_{1}$ are not necessarily absolutely continuous, where $V$ is not necessarily of the trace class, and where the limits (9), when they exist, may be only partially isometric, have been investigated. In particular, see Aronszajn [1], Kato [9], [10], Kuroda [12], Rosenblum [15], and, especially in the physical context of scattering theory, Cook [2], Hack [6], Jauch [7], Jauch and Zinnes [8]. (In the physical theory, the operators $H_{0}, H_{1}$ and $V$ correspond to the free Hamiltonian, total Hamiltonian and interaction potential respectively, see [7] and [8]; the operators $U_{+}$ and $U_{-}$of (9) have been called half-scattering operators by Friedrichs [5], p. 233, and wave operators by Jauch [7], p. 137.) When (3) is assumed and $H_{0}$ and $V$ satisfy certain conditions, these results imply the existence of the operators $U_{+}^{\epsilon}$ and $U_{-}^{\epsilon}$, as strong limits defined by (4) and satisfying (5). However, $U^{\epsilon}=U_{+}^{\epsilon}, U_{-}^{\epsilon}$ now may not be analytic perturbations of the identity, (6), and so (7) and (8) may not hold. It will be shown in the present paper that under certain conditions relation (8), hence also (7) and (6), must not hold.

Let $H_{0}$ and $H_{1}$ denote the free and perturbed Hamiltonian one-dimensional wave mechanical operators

$$
H_{0}=-d^{2} / d x^{2}, \quad H_{1}=H_{0}+V(x),
$$

on the Hilbert space $L^{2}(-\infty, \infty)$. It will be shown, as a consequence of a result of Kuroda [12], that if $V=V(x)$ is non-negative, bounded, and "small" for large $x$ (condition (15)) then necessarily the strong limits of (9) exist as unitary operators, and that furthermore, as a consequence of [14], if also $V(x)$ is "not too small" (condition (16)), then the spectra of these operators are the entire unit circle $|z|=1$. Correspondingly, if $H_{1}^{\epsilon}$ is defined by (3), then (4) and (5) hold, and $s p\left(U_{+}^{\bullet}\right)$ and $s p\left(U_{-}^{\circ}\right)$ are, for each $\epsilon>0$, also the unit circle. In this case then, relation (8) fails to hold, and $U_{+}$and $U_{-}$surely cannot be of the form (6). Use will be made of the following

Lemma. On a Hilbert space let $H_{0}$ be a non-negative (not necessarily bounded) selfadjoint operator, $V$ a bounded non-negative self-adjoint operator, and suppose that $H_{0}$ and $H_{1}=H_{0}+V$ are unitarily equivalent, thus

$$
H_{0} \geqq 0, \quad 0 \leqq V \leqq \text { const. } I, \quad H_{1}=U H_{0} U^{*} \quad \text { (U, unitary). }
$$

If $f$ is any element of the Hilbert space for which $g=V^{1 / 2} f \neq 0$ and $g$ is in the domain of $H_{0}$, then

$$
\text { meas } \operatorname{sp}(U) \geqq 2 \pi\left[1+2\|f\|^{2}\left(H_{0} g, g\right) /\|g\|^{4}\right]^{-1} .
$$

The proof of the Lemma was given in [14]. 
2. Theorem. Let $H_{0}$ and $H_{1}$ be defined on $L^{2}(-\infty, \infty)$ by (11), and suppose that $V(x)$ is continuous on $-\infty<x<\infty$, satisfies

$$
0 \leqq V(x) \leqq \text { const. on }-\infty<x<\infty \text {, }
$$

and that

$$
\int_{-\infty}^{\infty} V(x) d x<\infty .
$$

Then $H_{0}$ and $H_{1}$ are absolutely continuous and the strong limits $U_{+}$and $U_{-}$of (9) exist as unitary operators which satisfy (10). If, in addition,

$$
\liminf _{b-a \rightarrow \infty}(b-a)^{-3} \int_{a}^{b} V^{-1}(x) d x=0,
$$

then the spectrum of each operator $U_{+}$and $U_{-}$is the entire unit circle $|z|=1$.

Condition (16) rules out, for example, $V(x)=0$, a function for which the assertion of the Theorem concerning the spectra of the associated operators $U_{+}$and $U_{-}$is surely false. In fact, by (9), $U_{+}=I$ and $U_{-}=I$, hence also, corresponding to (3), $U_{+}^{\epsilon}=I$ and $U_{-}=I$ for all $\epsilon$.

It is easily verified that an example of a function satisfying all the conditions (14)-(16) is furnished by $V(x)=|x|^{-c}, 1<c<2(c=$ const.), when $x$ is large, and where $V(x)$ is defined so as to be continuous and non-negative near $x=0$.

3. Proof of the theorem. It is known that $H_{0}$ is absolutely continuous with the spectrum $0 \leqq \lambda<\infty$ (see Weyl [18], Kodaira [11], Titchmarsh [16], p. 59). Relation (14) guarantees that the spectrum of $H_{1}$ is contained in the half-line $0 \leqq \lambda<\infty$, while (15) implies via, for instance, asymptotic formulas for the solutions of the equation $y^{\prime \prime}+(\lambda-V(x)) y=0$, that $H_{1}$ is absolutely continuous with the spectrum $0 \leqq \lambda<\infty$; see Wintner [17], p. 421. Relations (14) and (15) imply also that $V(x)$ is of class $L^{2}(-\infty, \infty)$. An application of a result of Kuroda [12] (see Theorem 3.1, pp. 438-439 and Theorem 5.1, p. 21; also a reference cited on p. 21 to T. Ikebe) then implies the existence of the strong limits (9) as unitary operators satisfying (10).

In order to complete the proof of the Theorem it will be shown that (16) implies

$$
\inf _{0}\left[\int_{-\infty}^{\infty}{g^{\prime}}^{2} d x \int_{-\infty}^{\infty} V^{-1}(x) g^{2} d x /\left(\int_{-\infty}^{\infty} g^{2} d x\right)^{2}\right]=0,
$$

where $g, g^{\prime \prime}$ (hence $g^{\prime}$ ) and $V^{-1 / 2} g$ belong to $L^{2}(-\infty, \infty)$. Since

$$
\left(H_{0} g, g\right)=-\int_{-\infty}^{\infty} g g^{\prime \prime} d x=\int_{-\infty}^{\infty} g^{\prime 2} d x,
$$

it is seen that (17) implies meas $s p\left(U_{-}\right)=2 \pi$ by virtue of (13). There remains then to verify (17).

To this end, consider the function $y=z(x)$ defined on $-\infty<x<\infty$ by $z(x)=$ $(b-a)^{-3 / 2}(x-a)$ for $a \leqq x \leqq(a+b) / 2, z(x)=z(a+b-x)$ for $(a+b) / 2 \leqq x \leqq b$, and $z(x)=0$ for $x$ outside the interval $a=x=b$. It is seen that $z(x)$ is continuous, has a piecewise continuous first derivative, and that

$$
\int_{-\infty}^{\infty} z^{2} d x=1 / 12, \quad \int_{-\infty}^{\infty}{z^{\prime}}^{2} d x=(b-a)^{-2},
$$


and

$$
\int_{-\infty}^{\infty} V^{-1} z^{2} d x=\int_{a}^{b} V^{-1} z^{2} d x \leqq \int_{a}^{b} V^{-1} d x / 4(b-a) .
$$

Hence, the expression [ $\cdots]$ of (17) is, for $g=z$, majorized by const. $(b-a)^{-3} \int_{a}^{b} V^{-1} d x$. It is clear that each $z$ can be smoothed out so as to obtain a function $g$ possessing continuous second derivatives of the type allowed in (17) and such that $[\cdots]$ has again the same majorant. Condition (16) now yields (17) and the proof of the Theorem is complete.

\title{
REFERENCES
}

1. N. Aronszajn, On a problem of Weyl in the theory of singular Sturm-Liouville equations, Am. J. Math. 79, 597-610 (1957)

2. J. M. Cook, Convergence to the Moller wave-matrix, J. Math. and Phys. 36, 82-87 (1957)

3. K. O. Friedrichs, Über die Spektralzerlegung eines Integraloperators, Math. Ann. 115, 249-272 (1938)

4. K. O. Friedrichs, On the perturbation of continuous spectra, Communs. Appl. Math. 1, 361-401 (1948)

5. K. O. Friedrichs, Mathematical aspects of the quantum theory of fields, Interscience Publ., New York, 1953.

6. M. N. Hack, On convergence to the Moller wave operators, Nuovo Cimento 9, 731-733 (1958)

7. J. M. Jauch, Theory of the scattering operator, Helv. Phys. Acta 31, 127-158 (1958)

8. J. M. Jauch and I. I. Zinnes, The asymptotic condition for simple scattering systems, Nuovo Cimento 11, 553-567 (1959)

9. T. Kato, On finite-dimensional perturbations of self-adjoint operators, J. Math. Soc. Japan 9, 239-249 (1957)

10. T. Kato, Perturbation of continuous spectra by trace class operators, Proc. Japan Acad. 33, 260-264 (1957)

11. K. Kodaira, The eigenvalue problem for ordinary differential equations of the second order and Heisenberg's theory of S-matrices, Am. J. Math. 71, 921-945 (1949)

12. S. T. Kuroda, On the existence and the unitary property of the scattering operator, Nuovo Cimento 12, 431-454 (1959)

13. C. R. Putnam, Commutators and absolutely continuous operators, Trans. Am. Math. Soc. 87, 513-525 (1958)

14. C. R. Putnam, Commutators, perturbations, and unitary spectra, Acta Mathematica (to appear)

15. M. Rosenblum, Perturbation of the continuous spectrum and unitary equivalence, Pacific J. Math. 7, 997-1010 (1957)

16. E. C. Titchmarsh, Eigenfunction expansions associated with second-order differential equations, Oxford, 1946

17. A. Winter, Small perturbations, Am. J. Math. 67, 417-430 (1945)

18. H. Weyl, Über gewöhnliche Differentialgleichungen mit Singularitäten und die zugehörigen Entwicklungen willkürlicher Funktionen, Math. Ann. 68, 220-269 (1910)

\section{AN UPPER BOUND ON NON-NEGATIVE TRANSIENT RESPONSES*}

\author{
BY
}

\section{A. H. ZEMANIAN (New York University)}

In a recent note [1], it was shown that, if the real-valued function $w(t)$ of the real variable $t$ is zero for $t<0$ and if its Laplace transform $W(s)$ is given by

$$
W(s)=\frac{a_{n} s^{n}+a_{n-1} s^{n-1}+\cdots+a_{0}}{s^{m}+b_{m-1} s^{m-1}+\cdots+b_{0}}=\frac{N(s)}{D(s)},
$$

where $m \geq 2 n$ and the real parts of the roots of the polynomial $D(s)$ are all non-positive,

${ }^{*}$ Received July 5, 1961. 